\title{
THE SYNTHESIS AND APPLICATION OF PHOSPHORUS-NITROGEN FLAME RETARDANT RETANNING AGENT
}

\section{SINTEZA ŞI APLICAREA UNUI AGENT DE RETĂBĂCIRE PE BAZĂ DE FOSFOR ŞI AZOT CU PROPRIETĂJI IGNIFUGE}

\author{
Jinwei ZHANG ${ }^{1,2}$, Fan CHENG ${ }^{3}$, Zhengwu $\mathrm{Al}^{1,2}$, Wuyong $\mathrm{CHEN}^{1,2^{*}}$ \\ ${ }^{1}$ Key Laboratory of Leather Chemistry and Engineering of Ministry of Education, Sichuan University, Chengdu 610065, China \\ ${ }^{2}$ National Engineering Laboratory for Clean Technology of Leather Manufacture, Sichuan University, Chengdu 610065, China \\ ${ }^{3}$ Haining Leather Research Institute, Haining 314400
}

THE SYNTHESIS AND APPLICATION OF PHOSPHORUS-NITROGEN FLAME RETARDANT RETANNING AGENT

ABSTRACT. In order to improve the fire resistance of leather to meet the higher fire-safety requirement, a phosphorus-nitrogen flame retardant retanning agent (MTHPS-U for short) was synthesized and used to treat leather. The vertical flame test, oxygen index test, smoke density, mechanical properties, thickness increase, and shrinkage temperature were used to evaluate the properties of M-THPS-U, and the dosage of M-THPS-U was optimized at the same time. Furthermore, the hide powder treated with M-THPS-U was used to study the flame resistance effect by thermogravimetry (TG) and microscopy. The results showed that the fire resistance of leather was improved obviously by using $5 \%$ of M-THPS-U. With the increase of M-THPS-U dosage, the mechanical properties dropped, but the fire resistance, thickness increase and shrinkage temperature of the leather raised. The TG results indicated that the fire retardant could promote the fire resistance of leather by accelerating leather fiber converting into char and decreasing the temperature at break down. In short, not only does M-THPS-U improve the fire resistance of leather, but it also has retanning and filling effects.

KEY WORDS: leather, phosphorus-nitrogen flame retardant, retanning

SINTEZA ŞI APLICAREA UNUI AGENT DE RETĂBĂCIRE PE BAZĂ DE FOSFOR ŞI AZOT CU PROPRIETĂJI IGNIFUGE

REZUMAT. Pentru a îmbunătăţi rezistenţa la foc a pielii în vederea îndeplinirii cerinţelor din ce în ce mai stricte de siguranţă în caz de incendiu, s-a sintetizat un agent de ignifugare pe bază de fosfor-azot cu efect de retăbăcire (prescurtat M-THPS-U) şi s-a utilizat pentru a trata pielea. Proprietăţile M-THPS-U au fost evaluate prin încercarea verticală la flacără, testul indicelui de oxigen, densitatea fumului, testarea proprietăţilor mecanice, creşterea grosimii şi temperatura de contracţie, în acelaşi timp optimizându-se şi doza de M-THPS-U. Mai mult decât atât, pulberea de piele tratată cu M-THPS-U a fost utilizată pentru a studia efectul de rezistenţă la flacără prin termogravimetrie (TG) şi microscopie. Rezultatele au arătat că rezistenţa la foc a pielii a fost în mod evident îmbunătăţită utilizând M-THPS-U în proporţie de 5\%. Odată cu creşterea dozei de M-THPS-U, proprietăţile mecanice au scăzut, însă rezistenţa la foc, creşterea grosimii şi temperatura de contracţie a pielii au crescut. Rezultatele TG au indicat că agentul de ignifugare a conferit pielii rezistenţă la foc prin accelerarea transformării fibrelor de piele în carbon şi scăderea temperaturii la descompunere. Pe scurt, M-THPS-Uîmbunătăţeşte rezistenţa la foc a pielii, dar are şi efecte de retăbăcire şi de umplere.

CUVINTE CHEIE: piele, agent de ignifugare pe bază de fosfor-azot, retăbăcire

\section{LA SYNTHÈSE ET L'APPLICATION D'UN AGENT DE RETANNAGE À BASE DE PHOSPHORE-AZOTE AVEC DES PROPRIÉTÉS IGNIFUGES}

RÉSUMÉ. Afin d'améliorer la résistance du cuir au feu pour répondre à les plus grandes exigences de sécurité-incendie, on a synthétisé et utilisé un agent ignifuge de phosphore et d'azote aux effets retannage (en abrégé M-THPS-U) pour traiter le cuir. Les propriétés de M-THPS-U ont été évaluées par l'essai à la flamme verticale, la détermination de l'indice d'oxygène, la densité de fumée, les propriétés mécaniques, la croissance en épaisseur, la température de rétraction, en optimisant en même temps le dosage de M-THPS-U. En outre, la poudre de peau traitée avec M-THPS-U a été utilisée pour étudier l'effet ignifuge par la thermogravimétrie (TG) et par la microscopie. Les résultats ont montré que la résistance au feu du cuir a été évidemment améliorée en utilisant $5 \%$ de M-THPS-U. Avec l'augmentation de la dose de M-THPS-U, les propriétés mécaniques ont diminué, mais la résistance au feu, la croissance en épaisseur et la température de rétraction du cuir ont augmenté. Les résultats de TG ont indiqué que l'agent ignifuge favorise la résistance au feu du cuir en accélérant la conversion des fibres de cuir en charbon et en diminuant la température de décomposition. En bref, M-THPS-U non seulement améliore la résistance au feu de cuir, mais il a aussi des effets de retannage et de remplissage.

MOTS CLÉS: cuir, agent ignifuge de phosphore et d'azote, retannage

\footnotetext{
"Correspondence to: Wuyong CHEN, Key Laboratory of Leather Chemistry and Engineering of Ministry of Education, Sichuan University, Chengdu 610065, China, email: wuyong.chen@163.com
} 


\section{INTRODUCTION}

Leather has a better fire resistance than cloth and plastic materials in normal situations. The oxygen index of leather is between $21 \%$ and $27 \%$, so leather is a selfextinguishing material. However, the flameless combustion time of leather is long and there is a lot of smoke together with nasty smell during burning. The demands of leather for fire-safety are increasing, especially for upholstery, aircraft and automobile leather for which the risks of fire are very real, so the leather must be treated by fire retardant to improve flame resistance [1].

In order to make high-quality fire resistance leather, the influence of tanning agent, fatliquoring agent and finishing agent on the flammability of leather was studied [2-5]. A lot of flame retardants have been synthesized and applied to improve fire resistance of leather, especially phosphorus-nitrogen flame retardant, because the phosphorus and nitrogen have a synergistic effect on fire resistance, which has been a hot area of research in recent years [6-8]. Furthermore, the additional functions such as retanning and fatliquoring had been given to the flame retardant without any negative effect on fire resistance, it would benefit from extending the use of flame retardant [9].

In this research, a phosphorus-nitrogen flame retardant retanning agent (M-THPS-U for short) was synthesized by tetrakis (hydroxymethyl) phosphonium sulfate (THPS), urea, formaldehyde and sodium bisulfite. Because there are two fire retardant elements in M-THPS-U, the flame resistance is excellent. Besides, there are a lot of active groups in M-THPS-U such as hydroxymethyl, which ensures good performance of retanning and filling effects.

\section{EXPERIMENTAL}

\section{Materials}

Shaved goat wet blue with average thickness of $1.0 \mathrm{~mm}$ was prepared in the lab using a common process. The chrome hide powder (chrome content was about $0.3 \%$ ) was from Tanning Chemical Lab of Chinese Academy of Forestry Sciences in Nanjing. Other chemicals used for leather processing were

\section{INTRODUCERE}

Pielea are o rezistenţă la foc mai bună decât materiale precum pânza şi plasticul în condiţii normale. Indicele de oxigen al pielii este între $21 \%$ şi $27 \%$, aşadar pielea este un material cu efect de autoextincţie. $\mathrm{Cu}$ toate acestea, timpul de combustie fără flacără al pielii este îndelungat şi emite mult fum, însoţit de un miros urât în timpul arderii. Cerinţele de rezistenţă la foc a pieilor sunt din ce în ce mai mari, în special pentru tapiţeria aeronavelor şi automobilelor, unde riscurile de incendiu sunt foarte reale, aşadar pielea trebuie să fie supusă unui tratament de ignifugare pentrua-iîmbunătăţi rezistenţa la flacără [1] .

Pentru a fabrica piele de înaltă calitate, cu rezistenţă la foc, s-a studiat influenţa agentului tanant, a agentului de ungere şi a agentului de finisare asupra inflamabilităţii pielii [2-5]. S-au sintetizat multe tipuri de materiale ignifuge şi s-au aplicat pentru a îmbunătăţi rezistenţa la foc a pielii, în special materiale pe bază de fosfor-azot, deoarece fosforul şi azotul au un efect sinergic asupra rezistenţei la foc, un domeniu de cercetare de mare interes în ultimii ani [6-8]. În plus, agentului de ignifugare i s-au adus funcţii suplimentare, cum ar fi capacitatea de retăbăcire şi ungere, fără niciun efect negativ asupra rezistenţei la foc, având beneficiul de a extinde utilizarea agentului de ignifugare [9].

În această lucrare de cercetare, s-a sintetizat un agent de ignifugare pe bază de fosfor-azot cu efect de retăbăcire (M-THPS-U pe scurt), având în compoziţie sulfat de tetrakis (hidroximetil) fosfoniu (THPS), uree, formaldehidă şi bisulfit de sodiu. Întrucât M-THPS-U conţine două elemente de ignifugare, rezistenţa la flacără este excelentă. În plus, M-THPS-U conţine multe grupări active, cum ar fi hidroximetilul, ceea ce duce la performanţe bune ale efectelor de retăbăcire şi umplere.

\section{PARTEA EXPERIMENTALĂ}

\section{Materiale}

Piei de capră wet blue fălţuite cu grosime medie de 1,0 mm s-au tăbăcit la nivel de laborator utilizând un proces obişnuit. Pulberea de piele cromată (conţinutul de crom a fost de aproximativ 0,3\%) a provenit de la Laboratorul Chimic de Tăbăcire al Academiei Chineze de Ştiinţe Silvice din Nanjing. Alte substanţe chimice utilizate pentru prelucrarea 
commercial grade, and reagents used for analysis and synthesis were research grade.

\section{Preparation of M-THPS-U}

An amount of $72 \mathrm{~g}$ urea and $81.3 \mathrm{~g}$ THPS were put into a round-bottom flask, then some distilled water was added into the system and the flask was stirred at $80^{\circ} \mathrm{C}$ for 2 hours. After cooling down to the room temperature, a $0.5 \mathrm{~mol} / \mathrm{L} \mathrm{NaOH}$ solution was used to adjust the $\mathrm{pH}$ to about 8.5. An amount of $59.1 \mathrm{~g}$ formaldehyde and some water were added, then the system was stirred at $65^{\circ} \mathrm{C}$ for 1 hour and reflux condensation was processed at the same time. Another $31.2 \mathrm{~g}$ sodium bisulphite was added again for $50 \mathrm{~min}$ to get the M-THPS-U. The M-THPS-U was colorless oily liquor and the solid content was about $45 \%$. The schematic reaction formula are shown below. pielii au fost de calitate comercială, iar reactivii utilizaţi pentruanaliză şi sinteză au fost de calitate analitică.

\section{Prepararea M-THPS-U}

Într-un balon cu fund rotund s-au introdus 72 g uree şi 81,3 g THPS, apoi s-a adăugat puţină apă distilată şi balonul a fost agitat la $80^{\circ} \mathrm{C}$ timp de 2 ore. După răcire la temperatura camerei, s-a utilizat o soluţie de $\mathrm{NaOH}$ de $0,5 \mathrm{~mol} / \mathrm{l}$ pentru a regla $\mathrm{pH}$-ul la aproximativ 8,5 . S-au adăugat $59,1 \mathrm{~g}$ formaldehidă şi puţină apă, apoi amestecul a fost agitat la $65^{\circ} \mathrm{C}$ timp de 1 oră, efectuând simultan şi condensarea. S-au mai adăugat 31,2 g bisulfit de sodiu, timp de 50 min pentru a obţine M-THPS-U. M-THPS-U a fost obţinut sub formă de lichior uleios incolor, iar conţinutul solid a fost de aproximativ $45 \%$. Formula de reacţie schematică este prezentată mai jos.

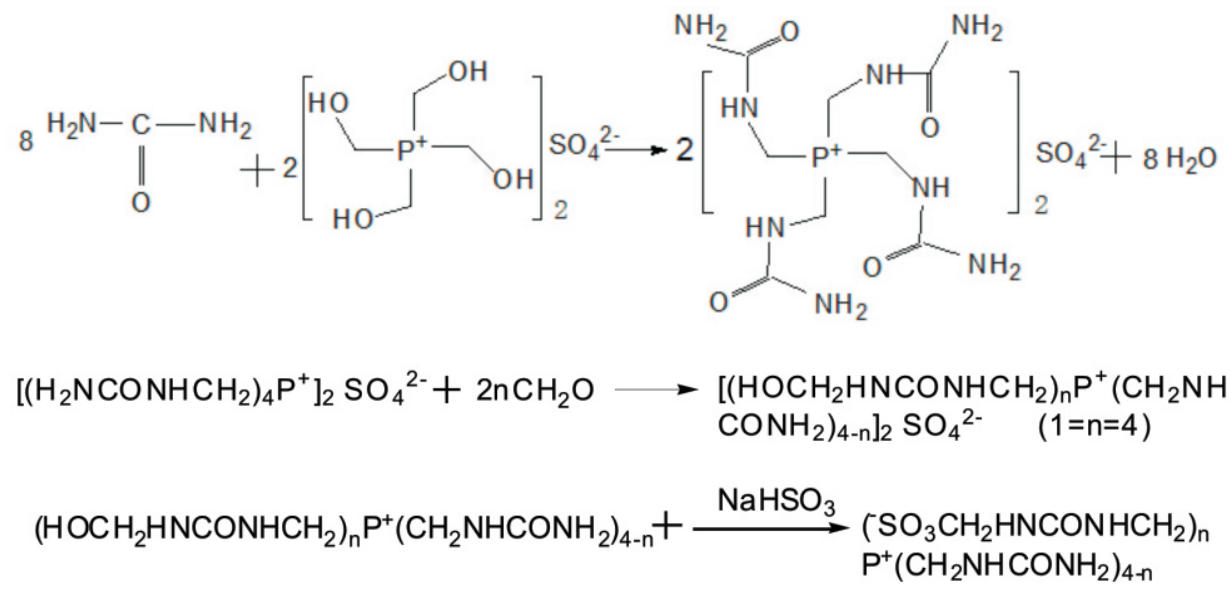

\section{Preparation of Fire Resistance Leather}

The shaved goat wet blue was treated by M-THPS$U$ with the process as shown in Table 1 and the treated leather was evaluated for flame retardance.

\section{Prelucrarea pielii rezistente la foc}

Pielea de capră wet blue fălţuită a fost tratată cu M-THPS-U utilizând procesul prezentat în Tabelul 1 şi sa testat pielea tratată pentru a determina rezistenţa la foc. 
Table 1: The process of flame retardant leather* Tabelul 1: Procesul de fabricare a pielii ignifuge*

\begin{tabular}{|c|c|c|c|c|c|}
\hline $\begin{array}{l}\text { Process } \\
\text { Proces }\end{array}$ & $\begin{array}{l}\text { Materials } \\
\text { Materiale }\end{array}$ & $\mathrm{T} /{ }^{\circ} \mathrm{C}$ & $\begin{array}{c}\text { Dosage, } \% \\
\text { Doză, \% }\end{array}$ & $\mathrm{t} / \mathrm{min}$ & $\begin{array}{c}\text { Remark } \\
\text { Observaţii }\end{array}$ \\
\hline \multirow{3}{*}{$\begin{array}{l}\text { Washing } \\
\text { Spălare }\end{array}$} & $\begin{array}{l}\text { Water } \\
\text { Apă }\end{array}$ & 35 & 200 & & \\
\hline & $\begin{array}{c}\text { Penetrant agent } \\
\text { Agent de penetrare }\end{array}$ & & 0.3 & 10 & \\
\hline & $\begin{array}{l}\text { Degreasing agent } \\
\text { Agent de degresare }\end{array}$ & & 0.2 & 20 & $\begin{array}{c}\text { Drain } \\
\text { Scurgere }\end{array}$ \\
\hline $\begin{array}{l}\text { Washing } \\
\text { Spălare }\end{array}$ & $\begin{array}{l}\text { Water } \\
\text { Apă }\end{array}$ & 25 & 200 & 10 & $\begin{array}{c}\text { Twice, drain } \\
\text { De două ori, scurgere }\end{array}$ \\
\hline \multirow{3}{*}{$\begin{array}{l}\text { Neutralizing } \\
\text { Neutralizare }\end{array}$} & $\begin{array}{l}\text { Water } \\
\text { Apă }\end{array}$ & 35 & 200 & & \\
\hline & HCOONa & & 0.5 & 20 & \\
\hline & $\mathrm{NaHCO}_{3}$ & & 0.9 & 60 & $\begin{array}{c}\mathrm{pH}: 6.0 \text {, drain } \\
p H: 6,0, \text { scurgere }\end{array}$ \\
\hline $\begin{array}{l}\text { Washing } \\
\text { Spălare }\end{array}$ & $\begin{array}{l}\text { Water } \\
\text { Apă }\end{array}$ & 25 & 200 & 15 & $\begin{array}{l}\text { Drain } \\
\text { Scurgere }\end{array}$ \\
\hline \multirow{3}{*}{$\begin{array}{l}\text { Fire retardant } \\
\text { Ignifugare }^{*}\end{array}$} & $\begin{array}{l}\text { Water } \\
\text { Apă }\end{array}$ & 35 & 150 & & \\
\hline & M-THPS-U & & X & 60 & \\
\hline & $\mathrm{HCOOH}$ & & 1.2 & & $\begin{array}{c}\mathrm{pH}: 4.0 \sim 4.2 \text {, drain } \\
\text { pH: 4,0 4,2, scurgere }\end{array}$ \\
\hline $\begin{array}{l}\text { Washing } \\
\text { Spălare }\end{array}$ & $\begin{array}{l}\text { Water } \\
\text { Apă }\end{array}$ & 25 & 200 & 10 & $\begin{array}{c}\text { Twice, drain } \\
\text { De două ori, scurgere }\end{array}$ \\
\hline $\begin{array}{l}\text { Drying } \\
\text { Uscare }\end{array}$ & & & & & \\
\hline
\end{tabular}

* The leather not treated with M-THPS-U was used as a control.

* Pielea netratată cu M-THPS-U a fost folosită ca martor.

\section{Preparation of Fire Resistant Hide Powder}

The hide powder (slightly chromed, chrome content was about $0.3 \%)$ and water $(500 \%$ water reported at hide powder weight) were mixed in a flask at room temperature for 4 hours to allow hide powder wetting. $10 \% \mathrm{M}-\mathrm{THPS}-\mathrm{U}$ (based on the solid content) was interacted with hide powder in thermostatic water bath oscillators at $30^{\circ} \mathrm{C}$ for 3 hours. After washing and filtration, the treated hide powder was dried in a vacuum drying oven at $40^{\circ} \mathrm{C}$ for 5 hours. The hide powder untreated by M-THPS-U was used as a control.

\section{Prepararea pulberii de piele ignifugate}

S-au amestecat pulberea de piele (uşor cromată, conţinutul de crom a fost de aproximativ $0,3 \%$ ) şi apa (500\% apă raportată la greutatea pulberii de piele) întrun balon, la temperatura camerei, timp de 4 ore, pentru a permite umezirea pulberii de piele. S-a interacţionat $10 \%$ M-THPS-U (pe baza conţinutului de materie solidă) cu pulberea de piele într-o baie de termostatare la $30^{\circ} \mathrm{C}$ timp de 3 ore. După spălare şi filtrare, pulberea de piele tratată s-a uscat într-un cuptor cu vid la $40^{\circ} \mathrm{C}$ timp de 5 ore. Pulberea de piele netratată cu M-THPS-U a fost utilizată ca probă martor. 


\section{Testing Methods}

\section{FT-IR of M-THPS-U}

The M-THPS-U was purified by absolute alcohol, and after drying in a vacuum drying oven, the white solid sample was obtained. The sample was ground with $\mathrm{KBr}$ and made into sheets, then a Nicolet10 FT-IR (American Thermo Scientific Corporation) was used to scan in the wavelength range of $400-4000 \mathrm{~cm}^{-1}$ for 32 times, and the data was recorded.

\section{TG of M-THPS-U and Hide Powder}

A NETZSCH TG 209 F1 thermogravimetric analyzer (Germany) was used for analysis of thermostability. The purified M-THPS-U was put into $\mathrm{Al}_{2} \mathrm{O}_{3}$ crucibles and heated at $10^{\circ} \mathrm{C} / \mathrm{min}$ in a $\mathrm{N}_{2}$ atmosphere (flow $\mathrm{N}_{2}: 100$ $\mathrm{mL} / \mathrm{min}$ ); the range of temperature was from 40 to $800^{\circ} \mathrm{C}$.

The treated and control hide powder were dried at $40^{\circ} \mathrm{C}$ for 24 hours. The dried hide powder was put into $\mathrm{Al}_{2} \mathrm{O}_{3}$ crucibles and heated at $10^{\circ} \mathrm{C} / \mathrm{min}$ in a $\mathrm{N}_{2}$ atmosphere (flow $\mathrm{N}_{2}: 100 \mathrm{~mL} / \mathrm{min}$ ); the range of temperature was from 40 to $800^{\circ} \mathrm{C}$. A NETZSCH TG 209 F1 thermogravimetric analyzer (Germany) was used for analysis of thermostability, TG and DTG curve recording.

\section{Carbon Residue Analysis}

The residue of hide powders after TG test were taken out of the crucible, and then a SBM-20TF optical microscope was used to observe the morphology of the carbon residue (100x).

\section{Flammability Test}

The vertical flame test was conducted in the CZF-3 horizontal-vertical tester produced by Nanjing Fangshan Analytical Equipment Factory according to ALCA Method E50 [10]. The oxygen index was measured according to the standard oxygen index test ASTM D 2863-77 [11] with a HC-2C instrument produced by Nanjing Fangshan Analytical Equipment Factory. According to GB/T 8627-1999 [12], a YM-3 building smoke density tester produced by Nanjing Fangshan Analytical Equipment Factory was used to test smoke density.

\section{Metode de testare}

\section{Analiza FT-IR a M-THPS-U}

M-THPS-U a fost purificat în alcool pur şi, după uscare într-un cuptor cu vid, s-a obţinut o probă solidă de culoare albă. Proba a fost măcinată cu $\mathrm{KBr}$ şi transformată în foi, apoi acestea s-au scanat utilizând un aparat FT-IR Nicolet10 (American Thermo Scientific Corporation) în intervalul de lungimi de undă 400-4000 $\mathrm{cm}^{-1}$ de 32 de ori şi s-au înregistrat datele.

\section{Analiza TG a M-THPS-U şi a pulberii de piele}

S-a utilizat un analizor termogravimetric NETZSCH TG 209 F1 (Germania) pentru analiza termostabilităţii. M-THPS-U purificat s-a introdus în creuzete din $\mathrm{Al}_{2} \mathrm{O}_{3}$ şi s-a încălzit la $10^{\circ} \mathrm{C} / \mathrm{min}$ într-o atmosferă de N2 (flux N2: $100 \mathrm{ml} / \mathrm{min}$ ); intervalul de temperatură a fost $4-80^{\circ} \mathrm{C}$.

Probele de pulbere de piele tratată şi martor s-au uscat la $40^{\circ} \mathrm{C}$ timp de 24 ore. Pulberea de piele uscată sa introdus în creuzete din $\mathrm{Al}_{2} \mathrm{O}_{3}$ şi s-a încălzit la $10^{\circ} \mathrm{C} / \mathrm{min}$ într-o atmosferă de N2 (flux N2: $100 \mathrm{ml} / \mathrm{min}$ ); intervalul de temperatură a fost $4-80^{\circ} \mathrm{C}$. S-a utilizat un analizor termogravimetric NETZSCH TG 209 F1 (Germania) pentru analiza termostabilităţii şi pentru înregistrarea curbelor TG şi DTG.

\section{Analiza reziduului de carbon}

Reziduul de pulbere de piele rezultat în urma testului TG a fost scos din creuzet, apoi s-a studiat cu un microscop optic SBM-20TF pentru a observa morfologia reziduului de carbon (100x).

\section{Testul de inflamabilitate}

Încercarea verticală la flacără s-a realizat în testerul orizontal-vertical CZF-3 produs de Nanjing Fangshan Analytical Equipment Factory conform metodei ALCA E50 [9]. Indicele de oxigen a fost măsurat în conformitate cu standardul de încercare al indicelui de oxigen ASTM D 2863-77 [11] cu un instrument HC-2C produs de Nanjing Fangshan Analytical Equipment Factory. Conform standardului GB/T 8627-1999 [12], sa utilizat un aparat de testare YM-3 produs de Nanjing Fangshan Analytical Equipment Factory pentru a testa densitatea fumului. 


\section{Mechanical Properties and Shrinkage Temperature}

(Ts) Test

The leathers were sampled [13] and conditioned [14] as the standard method. The tensile strength [15] and tear strength [16] of leathers were tested by a tensile machine (Al-7000S, China) following the standard method. The shrinkage temperature was tested by Shrinkage Temperature Tester (MSW-YD4, China) with the bath of glycerin (75\%). Each value was an average of two leather samples.

\section{Determinarea proprietăţilor mecanice şi a} temperaturii de contracţie (Ts)

S-au prelevat probele de piele [13] şi s-au condiţionat [14] utilizând metoda standard. S-au determinat rezistenţa la rupere [15] şi rezistenţa la sfâşiere [16] a pielii cu o maşină de tracţiune (AI-7000S, China) urmând metoda standard. Temperatura de contracţie a fost testată cu aparatul de testare a temperaturii de contracţie (MSW-YD4, China) cu baie de glicerină (75\%). Fiecare valoare a reprezentat media a două probe de piele.

\section{RESULTS AND DISCUSSION}

\section{REZULTATE ŞI DISCUTII}

\section{FT-IR of M-THPS-U}

\section{Analiza FT-IR a M-THPS-U}

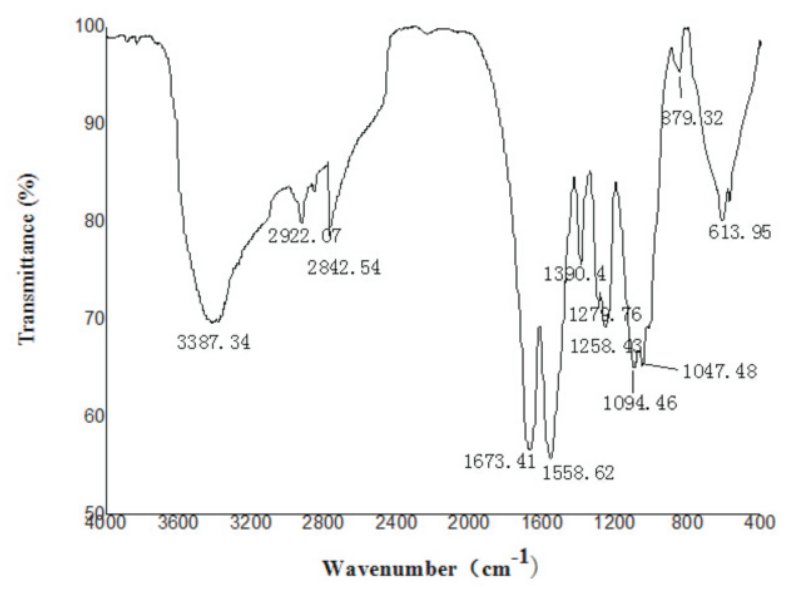

Figure 1. The FT-IR of M-THPS-U

Figura 1. Spectrul FT-IR al M-THPS-U

Figure 1 showed the main absorption wavelength of M-THPS-U. The peak at $1047 \mathrm{~cm}^{-1}$ was the $-\mathrm{CH}_{2} \mathrm{OH}$, which indicated the presence of hydroxymethyl in M-THPS-U. The peak at $3387 \mathrm{~cm}^{-1}$ was due to the $\mathrm{C}-\mathrm{N}$ stretching vibration of M-THPS-U, showing the urea acted with formaldehyde. The peak at $1258 \mathrm{~cm}^{-1}$ was the characteristic absorption peak of $-\mathrm{OH}$. The peak at $879 \mathrm{~cm}^{-}$ ${ }^{1}$ was due to the $\mathrm{S}=\mathrm{O}$ stretching vibration of M-THPS-U, indicating sulphonation reaction happening. All these results indicated that M-THPS-U had been synthesized as expected (see Preparation of M-THPS-U).
Figura 1 prezintă principalul spectru de absorbţie al M-THPS-U. Picul de la $1047 \mathrm{~cm}^{-1}$ a fost atribuit $-\mathrm{CH}_{2} \mathrm{OH}$, indicând prezenţa hidroximetilului în M-THPS-U. Picul de la $3387 \mathrm{~cm}^{-1} \mathrm{~s}$-a datorat vibraţiei de întindere C-N a MTHPS-U, arătând reacţia ureei cu formaldehida. Picul de la $1258 \mathrm{~cm}^{-1}$ este picul de absorbţie caracteristic al $-\mathrm{OH}$. Picul de la $879 \mathrm{~cm}^{-1} \mathrm{~s}$-a datorat vibraţiilor de întindere $\mathrm{S}=\mathrm{O}$ ale M-THPS-U, indicând producerea reacţiei de sulfonare. Toate aceste rezultate au indicat că M-THPS-U a fost sintetizat conform aşteptărilor (a se vedea Prepararea M-THPS-U). 
Table 2: The TG data of flame retardant and hide powder

Tabelul 2: Datele analizei TG ale agentului de ignifugare şi ale pulberii de piele

\begin{tabular}{|c|c|c|c|c|}
\hline $\begin{array}{c}\text { Sample } \\
\text { Probă }\end{array}$ & $\mathrm{T}_{10 \%} /{ }^{\circ} \mathrm{C}$ & $\mathrm{T}_{50 \%} /{ }^{\circ} \mathrm{C}$ & $\mathrm{CR}_{800^{\circ} \mathrm{C}} / \%$ & $\mathrm{~T}_{\max } /{ }^{\circ} \mathrm{C}$ \\
\hline M-THPS-U & 213.8 & 319.9 & 34.1 & 237.8 \\
\hline $\begin{array}{l}\text { Control hide powder } \\
\text { Pulbere piele martor }\end{array}$ & 227.3 & 353.1 & 18.5 & 327.3 \\
\hline $\begin{array}{l}\text { Treated hide powder } \\
\text { Pulbere piele tratată }\end{array}$ & 201.3 & 368.2 & 23.3 & $215.3 / 318.2$ \\
\hline
\end{tabular}

According to Table 2, the control hide powder was broken down rapidly at $327.3^{\circ} \mathrm{C}$ and its carbon residue was only $18.5 \%$ at $800^{\circ} \mathrm{C}$. However, the flame retardant hide powder had two rapid weight loss stages. They were $215.3^{\circ} \mathrm{C}$ and $318.2^{\circ} \mathrm{C}$, respectively, and the carbon residue was $23.3 \%$ at $800^{\circ} \mathrm{C}$. These results indicated that M-THPS- $U$ could increase the carbonization of collagen fiber, and decrease the weight loss. There was a linear relation between flame resistant effect and carbonization, the higher the carbonization, the better the flame resistant effect.

The $\mathrm{T}_{10 \%}, \mathrm{~T}_{50 \%}$ and $\mathrm{T}_{\max }$ of M-THPS- $\mathrm{U}$ were lower than the ones of hide powder (Table 2), so the M-THPS-U could break down before hide powder. This was the reason that the M-THPS-U could slow down the decomposition velocity of leather and enhance the thermal stability of fire resistant leather.

\section{Microscope Graph of Carbon Residue}

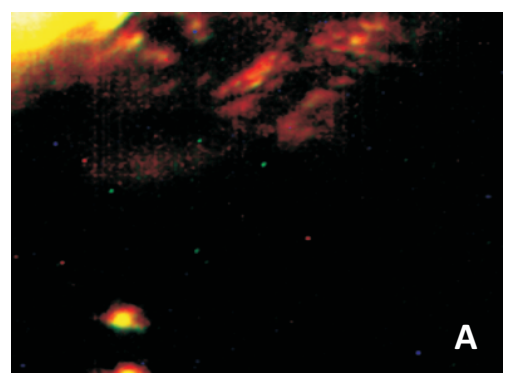

Conform Tabelului 2, proba martor de pulbere de piele s-a descompus rapid la $327,3^{\circ} \mathrm{C}$, iar reziduul de carbon a reprezentat doar $18,5 \%$ la $800^{\circ} \mathrm{C}$. Cu toate acestea, pulberea de piele ignifugată a avut două etape rapide de pierdere în greutate. Acestea au avut loc la $215,3^{\circ} \mathrm{C}$, respectiv la $318,2^{\circ} \mathrm{C}$, iar reziduul de carbon a reprezentat $23,3 \%$ la $800^{\circ} \mathrm{C}$. Aceste rezultate au indicat că M-THPS-U ar putea creşte carbonizarea fibrelor de colagen şi reduce pierderea în greutate. A existat o relaţie liniară între efectul de rezistenţă la foc şi carbonizare; cu cât a fost carbonizarea mai puternică, cu atât mai bun a fost efectul de rezistenţă la foc.

Valorile $\mathrm{T} 10 \%, \mathrm{~T} 50 \%$ şi $_{\text {max }}$ ale M-THPS-U au fost mai mici decât cele ale pulberii de piele (Tabelul 2), astfel încât M-THPS-U s-a descompus mai repede faţă de pulberea de piele. Acesta a fost motivul pentru care MTHPS-U ar putea încetini viteza de descompunere a pielii şi ar spori stabilitatea termică a pielii rezistente la foc.

\section{Imagine la microscop a reziduului de carbon}

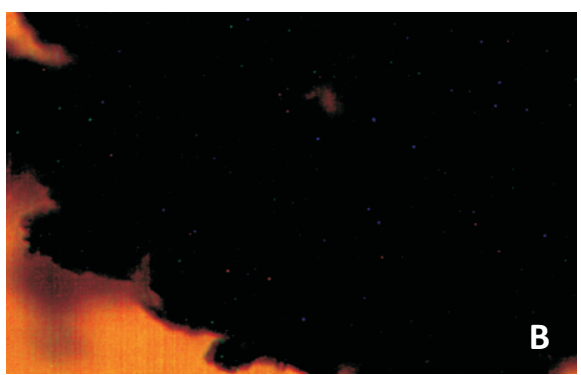

Figure 2. The char layer hide powder (A was control, B was flame-retarding hide powder)

Figura 2. Stratul carbonizat al pulberii de piele (A este proba martor, B este pulberea de piele ignifugată) 
The carbon residue was observed by optical microscope (Figure 2). Compared with the control, the char layer of hide powder treated with M-THPS-U was tight, indicating that M-THPS-U could improve the dehydration of fiber and carbonization. This effect had a barrier action which could prevent oxygen to play a role in leather combustion.

The Flammability of Fire-Resistance Leather
Reziduul de carbon a fost observat la microscopul optic (Figura 2). Comparativ cu proba martor, stratul carbonizat al pulberii de piele tratată cu M-THPS-U a fost îngust, indicând faptul că M-THPS-U ar putea îmbunătăţi deshidratarea fibrelor şi carbonizarea. Acest efect $a$ avut o acţiune de tip barieră care ar putea împiedica oxigenul să contribuie la combustia pielii.

\section{Inflamabilitatea pielii rezistente la foc}

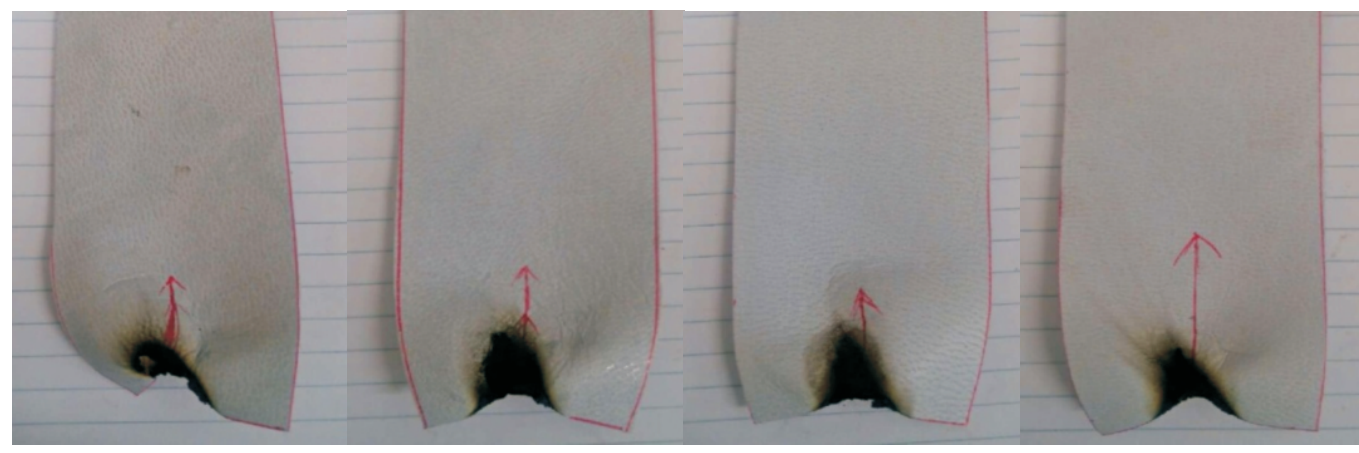
(a) $0 \%$ M-THPS-U
(b) $3 \%$ M-THPS-U
(c) $5 \%$ M-THPS-U
(d) $7 \%$ M-THPS-U

Figure 3. The state of treated leather after combustion

Figura 3. Starea pielii tratate după combustie

According to Figure 3, the leathers treated with MTHPS-U had larger combustion area and longer char length compared with control, so they showed a better fire resistance.
Conform Figurii 3, pieile tratate cu M-THPS-U au avut o zonă de ardere mai mare şi o dimensiune mai mare a zonei carbonizate comparativ cu proba martor, aşadar au prezentat o mai bună rezistenţă la foc.

Table 3: The vertical combustion of leather with dosage of M-THPS-U Tabelul 3: Combustia verticală a pielii în funcţie de doza de M-THPS-U

\begin{tabular}{|c|c|c|c|c|c|c|c|}
$\begin{array}{c}\text { Dosage of } \\
\text { M-THPS-U } \\
\text { Doza de M- } \\
\text { THPS-U }\end{array}$ & $\begin{array}{c}\text { Flame } \\
\text { combustion (s) } \\
\text { Ardere cu } \\
\text { flacără (s) }\end{array}$ & $\begin{array}{c}\text { Flameless } \\
\text { combustion (s) } \\
\text { Ardere fără } \\
\text { flacără }(s)\end{array}$ & $\begin{array}{c}\text { Char length }(\mathrm{cm}) \\
\text { Dimensiunea } \\
\text { zonei carbonizate } \\
(\mathrm{cm})\end{array}$ & $\begin{array}{c}\text { Weight loss } \\
(\%)\end{array}$ & $\begin{array}{c}\text { Oxygen index } \\
\text { Pierderea în } \\
\text { greutate (\%) }\end{array}$ & $\begin{array}{c}\text { Smoke density } \\
\text { Indicele de } \\
\text { oxigen (\%) }\end{array}$ & $\begin{array}{c}\text { Densitatea } \\
\text { fumului (\%) }\end{array}$ \\
\hline $0 \%$ & 2.68 & 16.48 & 0.89 & 4.19 & 27.5 & 54 \\
\hline $3 \%$ & 1.06 & 0 & 0.64 & 3.32 & 30.6 & 48 \\
\hline $5 \%$ & 0 & 0 & 0.56 & 3.08 & 32.1 & 45 \\
\hline
\end{tabular}

As shown in Table 3, with the increase of $M$ THPS-U, the flame combustion, flameless combustion, char length and weight loss of the leather gradually decreased and the oxygen index of the treated leathers gradually increased. When the
Aşa cum este prezentat în Tabelul 3, odată cu creşterea M-THPS-U, arderea cu flacără, arderea fără flacără, dimensiunea zonei carbonizate şi pierderea în greutate a pielii au scăzut progresiv, iar indicele de oxigen al pieilor tratate a crescut treptat. La o doză de 
dosage of M-THPS-U was $5 \%$, the flame combustion, flameless combustion were zero second, the char length and the weight loss were $0.56 \mathrm{~cm}$ and $3.08 \%$, which decreased by $37 \%$ and $26 \%$ compared with control, respectively. These properties meet the demand for the flammability of forest fire resistance glove leather [17].

Compared with control, the oxygen index of the leather increased by $11 \% \sim 20 \%$ and the smoke density decreased with the dosage of M-THPS-U to 7\%. The collagen fiber might be dehydrated by the M-THPS-U and the generated carbon needed higher quantities of heat and oxygen during combustion, so the smoke density cut down and the oxygen index increased with the dosage of M-THPS-U.

The Mechanical Properties, Ts and Thickness of Leather
M-THPS-U de 5\%, arderea cu flacără şi arderea fără flacără au avut loc în secunda zero, dimensiunea zonei carbonizate şi pierderea în greutate au fost de $0,56 \mathrm{~cm}$, respectiv 3,08\%, scăzând cu $37 \%$, respectiv $26 \%$, comparativ cu proba martor. Aceste proprietăţi au îndeplinit cerinţele de rezistenţă la incendiu forestier pentru mănuşile din piele [17].

Comparativ cu proba martor, indicele de oxigen al pielii a crescut cu $11 \%$ 20\%, iar densitatea fumului a scăzut cu creşterea dozei de M-THPS-U la 7\%. M-THPS$\mathrm{U}$ a deshidratat fibra de colagen şi carbonul generat a avut nevoie de cantităţi mari de căldură şi oxigen în timpul arderii, aşadar densitatea fumului a scăzut şi indicele de oxigen a crescut odată cu creşterea dozei de M-THPS-U.

Proprietăţile mecanice, Ts şi grosimea pielii

Table 4: The mechanical properties, Ts, and thickness of leather Tabelul 4: Proprietăţile mecanice, Ts şi grosimea pielii
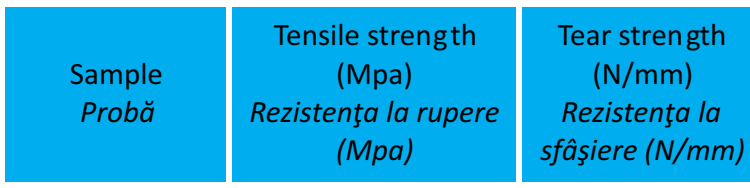

\begin{tabular}{|c|c|c|}
\hline $\begin{array}{c}\text { Control } \\
\text { Martor }\end{array}$ & 25.4 & 73.5 \\
\hline M-THPS-U 3\% & 23.1 & 63.7 \\
\hline M-THPS-U 5\% & 21.8 & 59.5 \\
\hline M-THPS-U 7\% & 19.7 & 57.5 \\
\hline
\end{tabular}

As shown in Table 4, the mechanical properties decreased with the dosage of M-THPS-U increasing, but the Ts and thickness increasing (\%) raised. M-THPS$U$ had many active groups such as hydroxymethyl which could act with collagen, so stress concentration was created by these cross-links between collagen fibers, which could lower the mechanical properties. The thickness reflected filling effect and the increasing of Ts expressed the tanning effect. The leather treated with M-THPS-U was thicker and had a higher Ts, showing that M-THPS-U had filling and tanning effect.

\section{CONCLUSIONS}

A phosphorus and nitrogen flame retardant (MTHPS-U) was synthesized for retanning leather with fire

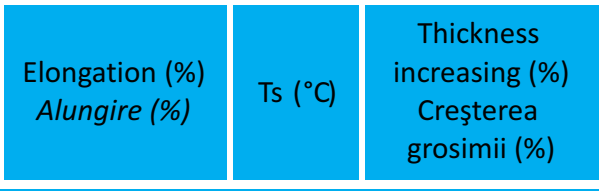

\begin{tabular}{|l|l|}
\hline 32.7 \\
\hline 33.4 \\
\hline 31.2 \\
\hline 30.6 \\
\hline
\end{tabular}

\begin{tabular}{|l|l|}
\hline 109.1 & 0 \\
\hline 112.8 & 6.3 \\
\hline 114.3 & 8.1 \\
\hline 113.7 & 8.9 \\
\hline
\end{tabular}

Aşa cum arată Tabelul 4, proprietăţile mecanice au scăzut cu creşterea dozei de M-THPS-U, însă Ts şi grosimea (\%) au crescut. M-THPS-U are multe grupări active, cum ar fi hidroximetilul, care a acţionat cu colagenul, astfel încât s-a creat o concentraţie de stres prin aceste legături încrucişate între fibrele de colagen, ceea ce a redus proprietăţile mecanice. Grosimea a reflectat efectul de umplere şi creşterea Ts a indicat efectul de tăbăcire. Pielea tratată cu M-THPS-U a fost mai groasă şi a avut Ts mai mare, ceea ce dovedeşte că M-THPS-U are efect de umplere şi de tăbăcire.

\section{CONCLUZII}

S-a sintetizat un agent de ignifugare pe bază de fosfor şi azot (M-THPS-U) pentru retăbăcirea pielii cu 
retardance. After treating with M-THPS-U, the smoke density, flame combustion, flameless combustion, char length and weight loss of leather decreased and oxygen index increased, indicating M-THPS- $U$ had excellent flame retardance. Also, the shrinkage temperature and thickness of leather increased, showing M-THPS-U had filling and tanning effect. To sum up, M-THPS-U could be used for making fire resistant leather with high performance.

\section{Acknowledgement}

The authors wish to thank the financial support of Science and Technology Department of Sichuan Province (Item No. 04GG009-021). efect de rezistenţă la foc. După tratarea cu M-THPS-U, densitatea fumului, arderea cu flacără, arderea fără flacără, dimensiunea zonei carbonizate şi pierderea în greutate a pielii au scăzut, iar indicele de oxigen a crescut, arătând că M-THPS-U are un efect de ignifugare excelent. De asemenea, temperatura de contracţie şi grosimea pielii au crescut, arătând că MTHPS-U are efect de umplere şi de tăbăcire. În concluzie, M-THPS-U ar putea fi folosit pentru a fabrica piele rezistentă la foc de înaltă calitate.

\section{Mulţumiri}

Autorii doresc să mulţumească pentru sprijin financiar Departamentului de Ştiinţă şi Tehnologie din Provincia Sichuan (nr. 04GG009-021).

\section{REFERENCES}

1. Ou, Y., Applied flame-retardant technology, Chemical Industry Publishing Company, Beijing, 2002.

2. Chen, W. et al., Influence of tanning on the flammability of leather, J. Soc. Leath. Tech. Chem., 2007, 91, 159-161.

3. Cheng, F., Jiang, L., Chen, W., Gaidau, C.C., Miu, L., Influence of Retanning Materials with Different Properties on the Flammability of Leather, Revista de Pielarie Incaltaminte (Leather and Footwear Journal), 2013, 13, 3, 179-186.

4. Huang, Z., Li, L., Chen, W., Influence of fatliquoring on the flammability of leather, J. Soc. Leath. Tech. Chem., 2006, 90, 155-158.

5. Gong, Y. et al., Influence of finishing on the flammability of leather, J. Soc. Leath. Tech. Chem., 2007, 91, 208-211.

6. Ling, H., Yang, J., Xiang, L. et al., The Synthesis and Application of a High Performance Amino Resin Nanocomposite as Leather Flame Retardant, J. Soc. Leath. Tech. Chem., 2012, 96, 5-10.

7. Wang, Q., Duan, B., Sun, G., Mechanism of Leather Flame Retardant and Selected of Flame Retardant Materials, Leather Science and Engineering Chem., 2006, 16, 4, 41-44.

8. Huang, Z., Synthesis of a Kind of Leather Flame Retardant, Sichuan University, China, 2005.

9. Huang, Z., Li, L., Chen, W., Latest Advances of Leather Flame Retardants and Leather Flame Retardant Techniques, Journal of Shaanxi University of Science \& Technology, 2004, 22, 3, 139- 142.

10. ALCA Method E 50. American Leather Chemists Association Method of Sampling and Analysis, 1984.

11. American Society for Testing Materials ASTM D 2863-77, ASTM Std 24, 1983, 262-269.

12. GB/T 8627-1999, Test method for density of smoke from the burning or decomposition of building materials, 1999, China.

13. IUP 2, Sampling. JSLTC, 2000, 84, 303-309.

14. IUP 3, Conditioning. JSLTC, 1998, 82, 199.

15. IUP 6, Measurement of tensile strength and percentage of elongation. JSLTC, 2000, 84, 317-321.

16. IUP 8, Measurement of tear load-double edge load. JSLTC, 2000, 84, 327-329.

17. LD59-94, Labor and labor security industry standard of the People's Republic of China, forest fire resistance glove, 1994, China.

Article received/Data primirii articolului: 29.06.2015

Accepted/Acceptat la data: 23.07.2015 